\title{
Precision determination of pion-nucleon coupling constants using effective field theory
}

\author{
P. Reinert, ${ }^{1}$ H. Krebs, ${ }^{1}$ and E. Epelbaum ${ }^{1}$ \\ ${ }^{1}$ Ruhr-Universität Bochum, Fakultät für Physik und Astronomie, \\ Institut für Theoretische Physik II, D-44780 Bochum, Germany
}

\begin{abstract}
The pion-nucleon coupling constants determine the strength of the long-range nuclear forces and play a fundamental part in our understanding of nuclear physics. While the charged- and neutral-pion couplings to protons and neutrons are expected to be very similar, owing to the approximate isospin symmetry of the strong interaction, the different masses of the up- and down-quarks and electromagnetic effects may result in their slightly different values. Despite previous attempts to extract these coupling constants from different systems, our knowledge of their values is still deficient. In this Letter we present a precision determination of these fundamental observables with fully controlled uncertainties from neutron-proton and proton-proton scattering data using chiral effective field theory. To achieve this goal, we use a novel methodology based on the Bayesian approach and perform, for the first time, a full-fledged partial-wave analysis of nucleon-nucleon scattering up to the pion production threshold in the framework of chiral effective field theory, including a complete treatment of isospin-breaking effects and our own determination of mutually consistent data. The resulting values of the pion-nucleon coupling constants are accurate at the percent level and show no significant charge dependence. These results mark an important step towards developing a precision theory of nuclear forces and structure.
\end{abstract}

PACS numbers: 14.20.Dh,21.30.-x,13.75.Cs,12.39.Fe

The pion-exchange mechanism drives the low-energy interaction between protons and neutrons and is of utmost importance for our understanding of atomic nuclei that make up $99.9 \%$ of the visible universe and for answering big science questions such as the origin of the elements, the limits of nuclear stability, searches for physics beyond the Standard Model and physics of neutron stars. The interaction of a charged and neutral pion with protons (p) and neutrons (n) is characterized by three coupling constants $f_{\pi^{0} \mathrm{pp}}, f_{\pi^{0} \mathrm{nn}}$ and $f_{\pi^{ \pm} \mathrm{pn}}$, whose precise definitions will be given below. These three constants determine the strength of the long- and intermediate-range nuclear forces originating from exchange of virtual pions. Their precise knowledge with controlled uncertainties is, therefore, of fundamental importance for a quantitative understanding of nuclear physics.

While possible in principle, the $a b$ initio precision determination of the pion-nucleon $(\pi \mathrm{N})$ coupling constants from lattice quantum-chromodynamics (QCD) and quantumelectrodynamics (QED) calculations is presently out of reach [1]. Attempts have been made to extract $f_{\pi^{ \pm} \mathrm{pn}}$ from experimental data on pion-nucleon $(\pi \mathrm{N})$ scattering [2-4], pionic atoms [5, 6] and proton-antiproton scattering [7], but the best way to determine all three constants is by analyzing the abundance of proton-proton (pp) and neutron-proton (np) scattering data. However, previous studies along this line [8-12] relied on phenomenological models and offered no way of a reliable uncertainty quantification apart from estimating statistical errors.

In this Letter we determine the values of all three $\pi \mathrm{N}$ coupling constants from nucleon-nucleon $(\mathrm{NN})$ scattering data using the model-independent framework of chiral effective field theory (EFT) [13, 14]. This method has already been applied to NN scattering, and the EFT expansion of the NN force has been recently pushed to fifth order $\left(\mathrm{N}^{4} \mathrm{LO}\right)$ [15-17]. The crucial new aspects of the current investigation include: (i) For the first time, a determination of all three $\pi \mathrm{N}$ coupling constants and a partial wave analysis of NN data up to the pion production threshold including the determination of mutually consistent data are carried out in the framework of chiral EFT. We have taken into account all chargeindependence-breaking (CIB) and charge-symmetry-breaking (CSB) isospin-violating $\mathrm{NN}$ interactions up through $\mathrm{N}^{4} \mathrm{LO}$. This allowed us to achieve a statistically perfect description of mutually consistent pp and np scattering data in the framework of chiral EFT that is unprecedented in its precision.

(ii) We have succeeded in overcoming the computational challenge of performing a Bayesian determination of the $\pi \mathrm{N}$ coupling constants. Contrary to the computationally much less demanding frequentist methods used in all previous determinations [8,-12, 18], the Bayesian approach provides a rigorous way to calculate the joint conditional probability density of the $\pi \mathrm{N}$ coupling constants given $\mathrm{NN}$ data.

(iii) A careful uncertainty analysis, facilitated by the usage of Bayesian methods, is performed to estimate not only statistical errors in the calculated $\pi \mathrm{N}$ coupling constants, but also systematic uncertainties from the truncation of the EFT expansion and the choice of the highest energy of the included $\mathrm{NN}$ data - a feature lacking in the earlier determinations of these quantities.

Definitions of the $\pi N$ coupling constants.-Consider first the interaction of the nucleon with the isovector weak current $A_{i}^{\mu}$, which is described in terms of the axial and induced pseudoscalar form factors $G_{A}$ and $G_{P}$. In the limit of exact isospin symmetry corresponding to the equal masses of the up- and down-quarks and in the absense of electromagnetic interactions, the matrix element of $A_{i}^{\mu}(x=0)$ between nucleon states can be parametrized via

$$
\left\langle\mathrm{N}\left(p^{\prime}\right)\left|A_{i}^{\mu}(0)\right| \mathrm{N}(p)\right\rangle=\bar{u}\left(p^{\prime}\right)\left[\gamma^{\mu} G_{A}+\frac{q^{\mu}}{2 m_{\mathrm{N}}} G_{P}\right] \gamma_{5} \frac{\tau_{i}}{2} u(p),
$$

with $u(p)$ and $\bar{u}\left(p^{\prime}\right)$ the corresponding Dirac spinors, $\tau_{i}$ 
the isospin Pauli matrices and $m_{\mathrm{N}}$ the nucleon mass. Furthermore, $q^{\mu}=\left(p^{\prime}-p\right)^{\mu}$ refers to the momentum transfer of the nucleon. The form factors $G_{A}\left(q^{2}\right)$ and $G_{P}\left(q^{2}\right)$ carry important information about the internal structure of the nucleon. For example, the axial charge of the nucleon $g_{A} \equiv G_{A}(0)=1.2756(13)$ [19] controls the decay rate of a neutron to a proton. Recently, this quantity was calculated from first principles at a per-cent level using lattice lattice quantum-chromodynamics (QCD) [20], see also [21] for a review of lattice QCD calculations of $g_{A}$. While $G_{A}\left(q^{2}\right)$ is a smooth function near $q^{2}=0$, the induced pseudoscalar form factor possesses a pion-pole contribution, $G_{P}\left(q^{2}\right)=$ $4 m_{\mathrm{N}} g_{\pi \mathrm{NN}} F_{\pi} /\left(M_{\pi}^{2}-q^{2}\right)+$ non-pole terms, whose residue is determined by the (pseudoscalar) $\pi \mathrm{N}$ coupling constant $g_{\pi \mathrm{NN}}$. The pion decay constant $F_{\pi}=(92.1 \pm 0.8) \mathrm{MeV}$ [19] determines the rate of weak decays $\pi^{ \pm} \rightarrow \mu^{ \pm} \nu_{\mu}$. The stronginteraction constant $g_{\pi \mathrm{NN}}$ is connected to $g_{A}$ and $F_{\pi}$ entering weak processes via the celebrated Goldberger-Treiman relation $F_{\pi} g_{\pi \mathrm{NN}}=g_{A} m_{\mathrm{N}}\left(1+\Delta_{\mathrm{GT}}\right)$, where the small Goldberger-Treiman discrepancy $\Delta_{\mathrm{GT}}$ is driven by the nonvanishing masses of the up- and down-quarks.

Away from the isospin limit and in the presence of quantum-electrodynamics (QED), one has to distinguish between protons and neutrons and between the charged and neutral pions by introducing three coupling constants $g_{\pi^{0} \mathrm{pp}}$, $g_{\pi^{0} \mathrm{nn}}$ and $g_{\pi^{ \pm} \mathrm{pn}}$ or, equivalently, the corresponding pseudovector couplings $f_{\mathrm{p}} \equiv f_{\pi^{0} \mathrm{pp}}=M_{\pi^{ \pm}} g_{\pi^{0} \mathrm{pp}} /\left(2 \sqrt{4 \pi} m_{\mathrm{p}}\right)$, $f_{\mathrm{n}} \equiv f_{\pi^{0} \mathrm{nn}}=M_{\pi^{ \pm}} g_{\pi^{0} \mathrm{nn}} /\left(2 \sqrt{4 \pi} m_{\mathrm{n}}\right)$ and $f_{\mathrm{c}} \equiv f_{\pi^{ \pm} \mathrm{pn}}=$ $M_{\pi^{ \pm}} g_{\pi^{ \pm} \mathrm{pn}} /\left(\sqrt{4 \pi}\left(m_{\mathrm{p}}+m_{\mathrm{n}}\right)\right)$. The determination of these fundamental constants from NN scattering data is the main subject of this study.

Chiral EFT for nuclear forces. - We use chiral EFT, an effective field theory of QCD, to describe the low-energy interactions between two nucleons, and employ the resulting NN potential to extract the $\pi \mathrm{N}$ coupling constants from a combined Bayesian analysis of $\mathrm{np}$ and pp scattering data below the pion production threshold. Chiral EFT utilizes an expansion in powers of momenta and pion masses to describe interactions between pions and nucleons in a systematically improvable way [13, 14, 22]. The corresponding effective Lagrangian contains all possible terms compatible with the symmetries of QCD. The non-perturbative dynamics of QCD is encoded in the so-called low-energy constants (LECs), which control the strength of the interactions in the effective Lagrangian and can be determined from experiments or lattice QCD calculations. Chiral EFT has also been extended to include virtual photons. Fig. 1 shows examples of contributions to the NN force in chiral EFT. The most important terms at leading order (LO) include one-pion exchange (Fig. 1p) and contact interactions (Fig. 1e). Two-pion exchange (Fig. 1 d) and one-photon exchange (Fig. 19) start to contribute at nextto-leading order (NLO), while pion-photon exchange (Fig.11.) appears first at fourth order $\left(\mathrm{N}^{3} \mathrm{LO}\right)$.

In recent years, the chiral expansion of the $\mathrm{NN}$ force has been pushed to fifth order [15-17]. All relevant isospin-

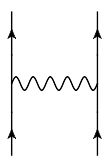

a

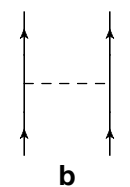

b

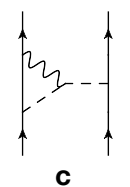

C

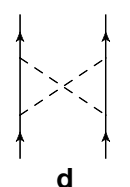

d

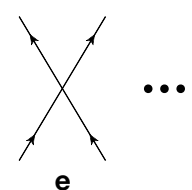

e
FIG. 1. Diagrammatic illustration of the NN interaction in chiral EFT. Photons, pions and nucleons are shown by wavy, dashed and solid lines, respectively. Diagrams $\mathbf{a}, \mathbf{b}, \mathbf{c}, \mathbf{d}$ and $\mathbf{e}$ are representative examples of the one-photon exchange, one-pion exchange (OPE), electromagnetic corrections to the OPE, two-pion exchange (TPE) and NN short-range contributions, respectively. The range of interactions decreases from the left to the right.

invariant $\pi \mathrm{N}$ LECs have been reliably determined from a dispersion theory analysis of $\pi \mathrm{N}$ scattering in Ref. [23]. Therefore, the long-range part of the $\mathrm{NN}$ interaction is parameterfree. To avoid distortions of the long-range forces due to a finite cutoff $\Lambda$, we introduced in Ref. [17] an improved local regulator which respects the analytic structure of the interaction. The LECs accompanying short-range operators (Fig. 1 1 e) were determined in Ref. [17] from a fit to the 2013 Granada database [24] of mutually compatible np and pp data. Furthermore, we have introduced a $\mathrm{N}^{4} \mathrm{LO}^{+} \mathrm{NN}$ potential, where the leading F-wave short-range interactions, formally appearing at sixth order, were taken into account in order to achieve a statistically satisfactory description of certain very precisely measured pp data, see also Ref. [16]. This allowed us to achieve a description of NN data on par with or even better than that based on the most precise phenomenological potentials, but with a much smaller number of adjustable parameters. However, the treatment of isospin-breaking (IB) effects in Ref. [17] was incomplete and limited to the one of the Nijmegen [25] and Granada 2013 [24] partial wave analyses (PWA).

In this Letter we include the charge-independence-breaking (CIB) and charge-symmetry-breaking (CSB) IB NN interactions complete up through $\mathrm{N}^{4} \mathrm{LO}$. In particular, we employ the most general form of the OPE potential including the leading electromagnetic corrections [26] and take into account the leading and subleading IB two-pion-exchange contributions [27-29]. These long-range interactions are expressed in terms of known LECs, the $\pi \mathrm{N}$ coupling constants $f_{\mathrm{p}}^{2}, f_{\mathrm{c}}^{2}$ and $f_{0}^{2} \equiv f_{\mathrm{p}} f_{\mathrm{n}}$ to be determined, the nucleon mass difference $\delta m=m_{\mathrm{n}}-m_{\mathrm{p}} \simeq 1.29 \mathrm{MeV}$ and its QCD contribution $\delta m^{\mathrm{QCD}}=2.05(30) \mathrm{MeV}$ [30], see Ref. [31] for an update and Ref. [32] for a recent $a b$ initio calculation using lattice QCD and QED. We also include short-range IB interactions in the ${ }^{1} \mathrm{~S}_{0},{ }^{3} \mathrm{P}_{0},{ }^{3} \mathrm{P}_{1}$ and ${ }^{3} \mathrm{P}_{2}$ partial waves. Details of the employed NN interaction are given in Supplemental Material.

Determination of the $\pi N$ coupling constants.-We end up with 33 parameters that need to be determined from NN data, comprising of $3 \pi \mathrm{N}$ LECs $f^{2} \equiv\left\{f_{\mathrm{c}}^{2}, f_{\mathrm{p}}^{2}, f_{0}^{2}\right\}$ and $25+5$ LECs $C_{i}$ from isospin-invariant + IB short-range interactions, collectively denoted as $C \equiv\left\{C_{i}\right\}$. For normally distributed 
errors, the likelihood of data $D$ given $f^{2}, C$ and $\Lambda$ is given by

$$
p\left(D \mid f^{2} C \Lambda\right)=\frac{1}{N} e^{-\frac{1}{2} \chi^{2}},
$$

where $N$ is a normalization constant. The data $D$ employed in our analysis include mutually compatible np and pp scattering data according to our own selection as detailed in Supplemental Material, where we also provide the definition of the $\chi^{2}$-measure. Using Bayes' theorem to relate the probability density function (PDF) $p\left(f^{2} C \Lambda \mid D\right)$ of the parameters given the data to $p\left(D \mid f^{2} C \Lambda\right)$, and integrating over the nuisance parameters $C$ and $\Lambda$, we obtain the quantity we are actually interested in, namely the PDF of $f^{2}$ given the data $D$

$$
p\left(f^{2} \mid D\right)=\int d \Lambda d C \frac{p\left(D \mid f^{2} C \Lambda\right) p\left(f^{2} C \Lambda\right)}{p(D)} .
$$

For the case at hand, $p(D)$ is a (normalization) constant. Furthermore, we use independent priors for $f^{2}, C$ and $\Lambda$ so that $p\left(f^{2} C \Lambda\right)=p\left(f^{2}\right) p(C) p(\Lambda)$, and employ a Gaussian prior for $C$ and uniform priors for $\Lambda$ and $f^{2}$ specified in Supplemental Material. To determine $f^{2}$ we need to find the maximum of $p\left(f^{2} \mid D\right)$ in Eq. (2). However, for each set of $f^{2}$, this requires integrating over a 31-dimensional space spanned by $\Lambda$ and $C$, which is not feasible. Instead, we employ the Laplace approximation by fitting $C$ to $D$ for fixed values of $f^{2}$ and $\Lambda$ and expressing the likelihood $p\left(D \mid f^{2} C \Lambda\right)$ as

$$
p\left(D \mid f^{2} C \Lambda\right) \approx \frac{1}{N} e^{-\frac{1}{2}\left[\chi_{\min }^{2}+\frac{1}{2}\left(C-C_{\min }\right)^{T} H\left(C-C_{\min }\right)\right]} .
$$

Here, $\chi_{\min }^{2} \equiv \chi_{\min }^{2}\left(f^{2}, \Lambda\right)$ at $C_{\min } \equiv C_{\min }\left(f^{2}, \Lambda\right)$ and the Hessian $H \equiv H\left(f^{2}, \Lambda\right)$ is given by $H_{i j}=\left.\frac{\partial^{2} \chi^{2}}{\partial C_{i} \partial C_{j}}\right|_{C=C_{\min }}$. Performing an analytical integration over $C$ then allows us to cast Eq. (2) into a numerically tractable form, see Supplemental Material for details. The remaining integration over $\Lambda \in[400,550] \mathrm{MeV}$ is performed numerically. We emphasize that reducing the amount of information in the employed priors for $C, f^{2}$ and $\Lambda$ has a negligible effect on our results, see Supplemental Material for details.

To account for the uncertainty inherent in the choice of the energy range of our PWA, we performed separate analyses of $\mathrm{NN}$ data up to the laboratory energies of $E_{\mathrm{lab}}^{\max }=220,240$, 260, 280 and $300 \mathrm{MeV}$. Furthermore, to address the systematic error stemming from the truncation of the EFT expansion for IB interactions, we considered two additional models of the NN interaction that include IB pion-photon- and two-pionexchange contributions beyond $\mathrm{N}^{4} \mathrm{LO}$. Our final PDF for the $\pi \mathrm{N}$ coupling constants are obtained by performing averaging over five values for $E_{\text {lab }}^{\max }$ and three models for IB interactions in order to account for the truncation uncertainty at $\mathrm{N}^{4} \mathrm{LO}$ as detailed in Supplemental Material. For all considered cases, the self-consistency of our results is verified by comparing the quantiles of the $\chi^{2}$ residuals with those of the assumed normal distribution (see Supplemental Material). Although no further assumptions regarding the shape of the distributions $p\left(f^{2} \mid D\right)$ have been made, the calculated PDFs $p\left(f^{2} \mid D\right)$ are found to

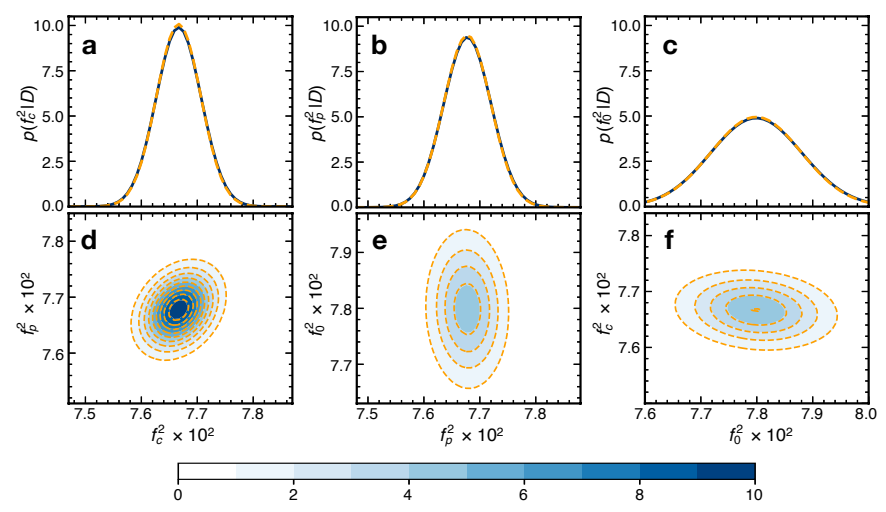

FIG. 2. Marginal posteriors for the central model and the energy range of $E_{\mathrm{lab}}=0-280 \mathrm{MeV}$. a, b and $\mathbf{c}$ show the probability distributions $p\left(f_{i}^{2} \mid D\right)$ in units of $10^{2}$. d, $\mathbf{e}$ and $\mathbf{f}$ show the joint distributions $p\left(f_{i}^{2}, f_{j}^{2} \mid D\right)$ in units of $10^{5}$. Blue solid lines and filled contours are based on the exact numerical evaluation, while orange dashed lines/contours represent its approximation by a multivariate Gaussian distribution as described in the text.

follow a multivariate Gaussian distribution to a very high accuracy. This is exemplified in Fig. 2 for the case of the central model and the energy range of $E_{\mathrm{lab}}=0-280 \mathrm{MeV}$. The distributions $p\left(f^{2} \mid D\right)$ can, therefore, be accurately characterized by the central values and errors of the $f_{i}^{2}$,s along with the corresponding correlation coefficients, which greatly facilitates their averaging as explained in Sec. 6 of the Supplementary Material.

We can verify the statistical validity of our results by studying the likelihood at the optimal values of the parameters. For example, fitting the LECs $C$ for $\Lambda=463.5 \mathrm{MeV}$ (see Fig. 1 of Supplemented Material) and the central values of the $f^{2}$, $s$ from Eq. (4) in the range of $E_{\text {lab }}=0-280 \mathrm{MeV}$ yields $\chi^{2}=$ 4950.72 for $N_{\text {dat }}=4926$, leading to $\chi^{2} / N_{\text {dat }}=1.005$. The quantity $\chi^{2} /\left(N_{\text {dat }}-N_{\text {par }}\right)-1=0.012$, where $N_{\text {par }}=34$ (including $\Lambda$ ), is comparable to half of the standard deviation (s.d.), $\sqrt{2 /\left(N_{\text {dat }}-N_{\text {par }}\right)}=0.020$, expected for a perfect model.

We have also investigated the robustness of our results with respect to the variation of input parameters. Specifically, the uncertainty from higher-order $\pi \mathrm{N}$ LECs entering the twopion-exchange potential is quantified by repeating our analysis for 50 sets of these LECs generated from the central values and covariance matrix of Ref. [23]. Furthermore, the uncertainty of the QCD contribution $\delta m^{\mathrm{QCD}}$, even taking its conservative estimate of $\pm 0.30 \mathrm{MeV}$ [30], is found to induce errors in $f_{i}^{2}$ that are negligibly small compared to the ones given below. Our final result for the $\pi \mathrm{N}$ coupling constants after the averaging reads

$$
\begin{aligned}
& f_{\mathrm{p}}^{2}=0.0770(5)^{\mathrm{a}}(0.8)^{\mathrm{b}}, \\
& f_{0}^{2}=0.0779(9)^{\mathrm{a}}(1.3)^{\mathrm{b}}, \\
& f_{\mathrm{c}}^{2}=0.0769(5)^{\mathrm{a}}(0.9)^{\mathrm{b}},
\end{aligned}
$$

where the first error (a) is obtained from the marginal posteriors $p\left(f^{2} \mid D\right)$ and includes the statistical and systematic errors 


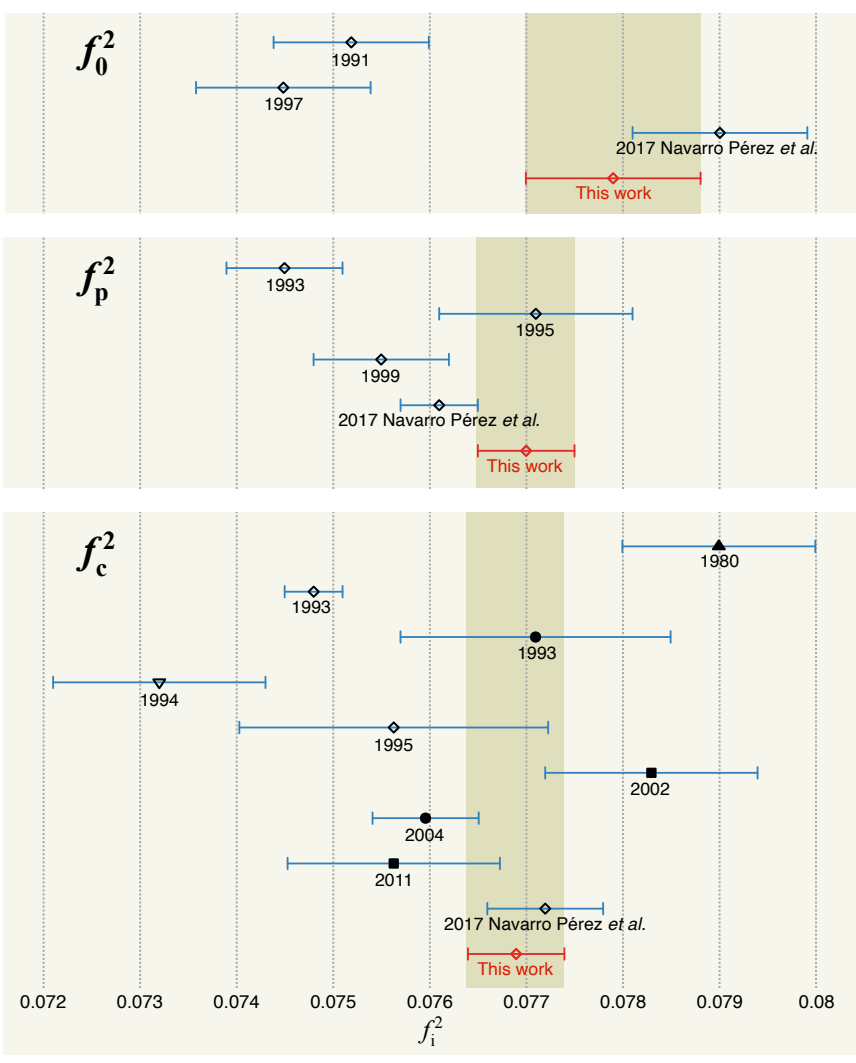

FIG. 3. Values for the $\pi \mathrm{N}$ coupling constants. The data points show selected determinations of the $\pi \mathrm{N}$ coupling constants $f_{0}^{2}, f_{\mathrm{p}}^{2}$ and $f_{\mathrm{c}}^{2}$. The results were obtained using $\pi \mathrm{N}$ PWA [2] (filled triangle), fixed- $t$ dispersion relations of $\pi \mathrm{N}$ scattering [3, 4] (filled dots), $\pi \mathrm{N}$ scattering lengths in combination with the GMO sum rule [5, 6] (filled squares), proton-antiproton PWA [7] (open triangle) and NN PWA [8, 11, 18] including the 2017 Granada PWA from Navarro Pérez et al. [12] (open diamonds). When provided separately, the statistical and systematic uncertainties are added in quadrature. The vertical bands show our full uncertainty. Uncertainties are one s.d.

due to the truncation of the EFT expansion, the choice of the energy range and the associated data selection. The second error (b) reflects the uncertainty in the higher-order $\pi \mathrm{N}$ LECs. Notice that the present study can, in principle, be extended to obtain a joint posterior probability distribution for $f_{i}$ 's and the higher-order $\pi \mathrm{N}$ LECs that can be useful for uncertainty quantification in chiral EFT via a combined analysis of the NN data and the experimental/empirical information on the $\pi \mathrm{N}$ scattering amplitude, see Ref. [33] for a related work.

Discussion of the results.-Our results for $f_{i}^{2}$ are compared in Fig. 3 with selected earlier determinations. Similarly to the Granada PWA, we find considerably larger values for the coupling constants as compared to the ones recommended by the Nijmegen group [9]. As already found in Ref. [12], this difference cannot be explained by the new experimental data since 1993 (see Supplemental Material for more details), thus pointing towards a possible sizeable systematic uncertainty from the interaction modeling in the Nijmegen PWA. Our value for $f_{\mathrm{c}}^{2}$ is consistent with the determinations from the $\pi \mathrm{N}$ system in Refs. [3-6] (at the $1.3 \sigma$ level). The results for $f_{0}^{2}$ and $f_{\mathrm{c}}^{2}$ agree within errors with the recent determination by the Granada group [12], while for $f_{\mathrm{p}}^{2}$ we obtain a slightly larger value. However, contrary to the Granada group that found evidence that the coupling of neutral pions to neutrons is larger than to protons, $f_{0}^{2}-f_{\mathrm{p}}^{2}=0.0029(10)$ [12], our result $f_{0}^{2}-f_{\mathrm{p}}^{2}=0.0010(10)^{\mathrm{a}}(2)^{\mathrm{b}}$ is consistent with no charge dependence. This difference may point to significant systematic uncertainties in the analysis by the Granada group [12] which are not quantified in that paper, in particular due to the cutoff radius $r_{c}$ and phenomenological modeling of the interaction. In contrast, our analysis relies on the systematically improvable EFT framework and takes into account modelindependent long- and intermediate-range nuclear interactions due to exchange of virtual pions and photons. This allows us to substantially reduce the number of adjustable parameters (33 in our analysis versus 55 in Ref. [12]) while still achieving at least a comparable description of NN data below the pion production threshold. Compared to the Granada analysis, we also do not find a large anticorrelation between $f_{0}^{2}$ and $f_{\mathrm{c}}^{2}$, see Table V of Supplemental Material.

In summary, our Bayesian determination of the $\pi \mathrm{N}$ coupling constants from $n p$ and pp scattering data in the framework of chiral EFT yields new reference values for these fundamental observables, accurate at the percent level. It provides new insights into the isospin symmetry of the strong interaction at the hadronic level by quantifying the charge dependence of these quantities. Our work also establishes important benchmarks for future first principles calculations using lattice QCD and QED, see [32] for a first step along this line, and opens the door for precision studies of nuclear structure and reactions by fixing the strengths of the longrange nuclear forces. As a very recent example, we mention the high-accuracy calculation of the deuteron charge and quadrupole form factors [34], where the isospin-breaking corrections considered in this work have been taken into account and were found to play an important role for the determination of the quadrupole moment, for which the value of $Q_{d}=0.2854_{-0.0017}^{+0.0038} \mathrm{fm}^{2}$ was obtained. Redoing the same analysis with the IB corrections from this paper being switched off, i.e. using the NN interactions from [17], the central value of the quadrupole moment would change significantly to $Q_{d}=0.2803 \mathrm{fm}^{2}$. These calculations are currently being extended to other light nuclei, and we expect the considered IB corrections to be relevant at the desired accuracy level. It would also be interesting to explore the implications of our study for the understanding of charge symmetry breaking in binding energy differences of mirror nuclei [35] and for certain low-energy three-nucleon scattering observables such as the doublet scattering length [36] and vector analyzing power $A_{y}$ [37]. Furthermore, since our analysis also results in an accurate determination of the IB short-range operators in the ${ }^{1} \mathrm{~S}_{0}$ partial wave, it may shed new light on the ongoing studies of neutrinoless double-beta decay in chiral EFT, see Ref. [38] for a related discussion. This will be addressed in a separate publication. Last but not least, we emphasize that the value 
for $f_{c}$ can be related to the pionic hydrogen width $\Gamma_{1 s}$, whose measurement at PSI is currently being analyzed, see [39] for a preliminary result.

We are grateful to Martin Hoferichter, Ulf-G. Meißner and Enrique Ruiz Arriola for sharing their insights into the considered topics and useful discussions. We also thank Ulf-G. Meißner for helpful comments on the manuscript. This work was supported by BMBF (contract No. 05P18PCFP1) and by DFG through funds provided to the Sino-German CRC 110 "Symmetries and the Emergence of Structure in QCD" (Grant No. TRR110).

[1] G. S. Bali, L. Barca, S. Collins, M. Gruber, M. Löffler, A. Schäfer, W. Söldner, P. Wein, S. Weishäupl, and T. Wurm (RQCD), J. High Energy Phys 05, 126 (2020)

[2] R. Koch and E. Pietarinen, Nucl. Phys. A 336, 331 (1980)

[3] F. G. Markopoulou-Kalamara and D. V. Bugg, Phys. Lett. B 318, 565 (1993)

[4] R. A. Arndt, W. J. Briscoe, I. I. Strakovsky, R. L. Workman, and M. M. Pavan, Phys. Rev. C 69, 035213 (2004)

[5] T. E. O. Ericson, B. Loiseau, and A. W. Thomas, Phys. Rev. C 66, 014005 (2002)

[6] V. Baru, C. Hanhart, M. Hoferichter, B. Kubis, A. Nogga, and D. R. Phillips, Nucl. Phys. A 872, 69 (2011)

[7] R. Timmermans, T. A. Rijken, and J. J. de Swart, Phys. Rev. C 50, 48 (1994)

[8] R. A. M. Klomp, V. G. J. Stoks, and J. J. de Swart, Phys. Rev. C 44, R1258 (1991).

[9] V. G. J. Stoks, R. Timmermans, and J. J. de Swart, Phys. Rev. C 47, 512 (1993)

[10] D. V. Bugg and R. Machleidt, Phys. Rev. C 52, 1203 (1995)

[11] J. J. de Swart, M. C. M. Rentmeester, and R. G. E. Timmermans, PiN Newslett. 13, 96 (1997).

[12] R. Navarro Pérez, J. E. Amaro, and E. Ruiz Arriola, Phys. Rev. C 95, 064001 (2017)

[13] S. Weinberg, Phys. Lett. B 251, 288 (1990)

[14] E. Epelbaum, H.-W. Hammer, and U.-G. Meißner, Rev. Mod. Phys. 81, 1773 (2009)

[15] E. Epelbaum, H. Krebs, and U.-G. Meißner, Phys. Rev. Lett. 115, 122301 (2015)
[16] D. R. Entem, R. Machleidt, and Y. Nosyk, Phys. Rev. C 96, 024004 (2017)

[17] P. Reinert, H. Krebs, and E. Epelbaum, Eur. Phys. J. A 54, 86 (2018)

[18] M. C. M. Rentmeester, R. G. E. Timmermans, J. L. Friar, and J. J. de Swart, Phys. Rev. Lett. 82, 4992 (1999)

[19] P. A. Zyla et al. (Particle Data Group), Prog. Theor. Exp. Phys. 2020, 083C01 (2020)

[20] C. C. Chang et al., Nature 558, 91 (2018)

[21] S. Aoki et al. (Flavour Lattice Averaging Group), Eur. Phys. J. C 80, 113 (2020).

[22] V. Bernard, N. Kaiser, and U.-G. Meißner, Int. J. Mod. Phys. E 04, 193 (1995)

[23] M. Hoferichter, J. Ruiz de Elvira, B. Kubis, and U.-G. Meißner, Phys. Rev. Lett. 115, 192301 (2015)

[24] R. Navarro Pérez, J. E. Amaro, and E. Ruiz Arriola, Phys. Rev. C 88, 064002 (2013), [Erratum: Phys. Rev. C 91, 029901 (2015)].

[25] V. G. J. Stoks, R. A. M. Klomp, M. C. M. Rentmeester, and J. J. de Swart, Phys. Rev. C 48, 792 (1993)

[26] U. van Kolck, M. C. M. Rentmeester, J. L. Friar, J. T. Goldman, and J. J. de Swart, Phys. Rev. Lett. 80, 4386 (1998)

[27] J. L. Friar and U. van Kolck, Phys. Rev. C 60, 034006 (1999)

[28] J. L. Friar, U. van Kolck, G. L. Payne, and S. A. Coon, Phys. Rev. C 68, 024003 (2003)

[29] E. Epelbaum and U.-G. Meißner, Phys. Rev. C 72, 044001 (2005)

[30] J. Gasser and H. Leutwyler, Nucl. Phys. B 94, 269 (1975)

[31] J. Gasser, H. Leutwyler, and A. Rusetsky, Phys. Lett. B 814, 136087 (2021)

[32] S. Borsanyi et al., Science 347, 1452 (2015)

[33] B. D. Carlsson, A. Ekström, C. Forssén, D. F. Strömberg, G. R. Jansen, O. Lilja, M. Lindby, B. A. Mattsson, and K. A. Wendt, Phys. Rev. X 6, 011019 (2016)

[34] A. A. Filin, D. Möller, V. Baru, E. Epelbaum, H. Krebs, and P. Reinert, Phys. Rev. C 103, 024313 (2021)

[35] J. L. Friar, G. L. Payne, and U. van Kolck, Phys. Rev. C 71, 024003 (2005)

[36] H. Witała, A. Nogga, H. Kamada, W. Glöckle, J. Golak, and R. Skibinski, Phys. Rev. C 68, 034002 (2003)

[37] H. Witała, D. Hüber, and W. Glöckle, Phys. Rev. C 49, R14 (1994)

[38] V. Cirigliano, W. Dekens, J. de Vries, M. L. Graesser, E. Mereghetti, S. Pastore, M. Piarulli, U. van Kolck, and R. B. Wiringa, Phys. Rev. C 100, 055504 (2019)

[39] D. Gotta et al., Hyperfine Interact. 234, 105 (2015) 


\section{SUPPLEMENTAL MATERIAL}

\section{Chiral effective field theory for the $\mathrm{NN}$ potential}

The functional form of the NN interaction employed in our analysis corresponds to the $\mathrm{N}^{4} \mathrm{LO}^{+}$potential of Ref. [1], supplemented with the CIB and CSB interactions up to $\mathrm{N}^{4} \mathrm{LO}$ as described below.

- We employ the most general form of the static OPE potential given by

$$
V_{1 \pi}^{\mathrm{pp}}=f_{\mathrm{p}}^{2} V\left(M_{\pi^{0}}\right), \quad V_{1 \pi}^{\mathrm{nn}}=f_{\mathrm{n}}^{2} V\left(M_{\pi^{0}}\right), \quad V_{1 \pi}^{\mathrm{np}}=-f_{0}^{2} V\left(M_{\pi^{0}}\right)+(-1)^{I+1} 2 f_{\mathrm{c}}^{2} V\left(M_{\pi^{ \pm}}\right),
$$

where $I=0,1$ is the isospin quantum number of the np system and $V\left(M_{i}\right)$ is given by

$$
V\left(M_{i}\right)=-\frac{4 \pi}{M_{\pi^{ \pm}}^{2}} \frac{\vec{\sigma}_{1} \cdot \vec{q} \vec{\sigma}_{2} \cdot \vec{q}}{\vec{q}^{2}+M_{i}^{2}}
$$

Here, $\vec{\sigma}$ are Pauli spin matrices and $\vec{q}=\vec{p}^{\prime}-\vec{p}$ is the momentum transfer of the nucleons, with $\vec{p}^{\prime}$ and $\vec{p}$ denoting the final and inititial momenta.

- Electromagnetic corrections to the OPE start to contribute at $\mathrm{N}^{3} \mathrm{LO}$. The expressions for the corresponding $\pi \gamma$-exchange potential at this order have been derived in Ref. [2] and depend only on the known quantities $\alpha, F_{\pi}, g_{A}$ and the pion masses. We employ the convention to absorb the pion-pole contribution to the $\pi \gamma$-exchange potential into a definition of $f_{\mathrm{c}}^{2}$.

- At the order considered, the IB two-pion-exchange potential involves the following structures

$$
V_{2 \pi}=\left(V_{C}+V_{S} \vec{\sigma}_{1} \cdot \vec{\sigma}_{2}+V_{T} \vec{\sigma}_{1} \cdot \vec{q} \vec{\sigma}_{2} \cdot \vec{q}\right) \tau_{1}^{3} \tau_{2}^{3}+\left(W_{C}+W_{S} \vec{\sigma}_{1} \cdot \vec{\sigma}_{2}+W_{T} \vec{\sigma}_{1} \cdot \vec{q} \vec{\sigma}_{2} \cdot \vec{q}\right)\left(\tau_{1}^{3}+\tau_{2}^{3}\right),
$$

with the first and second lines showing the CIB and CSB operators, respectively. The leading IB TPE contributions appear at fourth order $\left(\mathrm{N}^{3} \mathrm{LO}\right)$. The corresponding unregularized expressions for $V_{C}^{(4)}$ and $W_{C, S, T}^{(4)}$ are given in Eqs. (3.40) and (3.47) of Ref. [3] with $\Lambda=\infty$, while $V_{T}^{(4)}=V_{S}^{(4)}=0$. The expressions at fifth order ( $\left.{ }^{4} \mathrm{LO}\right)$ are given in Eqs. (3.49), (3.52) of Ref. [3] for $V_{T, S}^{(5)}$ and in Eq. (2.11) of Ref. [4] for $W_{C}^{(5)}$. For the remaining IB TPE contributions at this order, we employ the expressions

$$
\begin{aligned}
W_{T}^{(5)}(q) & =-\frac{1}{q^{2}} W_{S}^{(5)}(q)=-\left(\frac{g_{A}^{2}}{16 \pi^{2} F_{\pi}^{4}} \delta m c_{4}+\frac{4 f_{\mathrm{c}}^{3}}{M_{\pi^{ \pm}}^{4}}\left(f_{\mathrm{p}}-f_{\mathrm{n}}\right)\right) L(q), \\
V_{C}^{(5)}(q) & =-\frac{f_{\mathrm{c}}\left(2 f_{\mathrm{c}}-f_{\mathrm{p}}-f_{\mathrm{n}}\right)}{24 \pi F_{\pi}^{2} M_{\pi^{ \pm}}^{4}}\left(\frac{16 \pi F_{\pi}^{2} f_{\mathrm{c}}^{2}}{4 M_{\pi}^{2}+q^{2}}\left(128 M_{\pi}^{4}+112 M_{\pi}^{2} q^{2}+23 q^{4}\right)-\left(8 M_{\pi}^{2}+5 q^{2}\right) M_{\pi^{ \pm}}^{2}\right) L(q),
\end{aligned}
$$

where $q \equiv|\vec{q}|, M_{\pi}=2 / 3 M_{\pi^{ \pm}}+1 / 3 M_{\pi^{0}}$ and the function $L(q)$ is given by $L(q)=s / q \ln \left((s+q) /\left(2 M_{\pi}\right)\right.$ with $s=$ $\sqrt{q^{2}+4 M_{\pi}^{2}}$. The above expressions generalize the ones of Ref. [3] by including all TPE terms linear in the differences between $f_{i}$ 's with no assumptions about the dominance of CSB over CIB.

The employed IB TPE depends on the known LECs $F_{\pi}, g_{A}, c_{1}, c_{2}, c_{3}, c_{4}$, pion masses, the neutron-proton mass differences $\delta m$ and $\delta m^{\mathrm{QCD}}$ and the IB $\pi \mathrm{N}$ coupling constants $f_{\mathrm{p}}, f_{\mathrm{n}}$ and $f_{\mathrm{c}}$ to be determined.

- Up to the considered order, the CIB and CSB short-range interactions contributing to the np and pp systems involve two terms in the ${ }^{1} \mathrm{~S}_{0}$ channel and one term in each of the ${ }^{3} \mathrm{P}_{0},{ }^{3} \mathrm{P}_{1}$ and ${ }^{3} \mathrm{P}_{2}$ partial waves, see Ref. [3] for explicit expressions. The corresponding LECs have to be extracted from NN data.

Regularization of the IB potential is performed in the same way as in Ref. [1]. In particular, contact interactions are multiplied with a nonlocal Gaussian cutoff, while regularization of the long-range contributions is carried out by utilizing the spectral representation. For the contributions to the $\pi \gamma$-exchange potential and $V_{C}^{(4)}$ that feature a pole at the branch point, the spectral representation is employed for the corresponding indefinite integral.

Having defined the functional form of the employed NN potential, we are now in the position to specify the numerical values of various parameters. The long-range interactions stemming from the pion, pion-photon and two-pion exchanges are completely determined by the $\pi \mathrm{N}$ coupling constants $f_{\mathrm{p}}^{2}, f_{0}^{2}$ and $f_{\mathrm{c}}^{2}$ treated as free parameters, the cutoff value $\Lambda$ to be marginalized over and the known masses and constants specified in Table S1 To account for the Goldberger-Treiman discrepancy, we replace 
TABLE S1. Parameters for the long-range part of the NN potential

\begin{tabular}{cr}
\hline Quantity & Employed value \\
\hline Masses and mass differences: & $139.57 \mathrm{MeV}$ \\
$M_{\pi^{ \pm}}$ & $134.98 \mathrm{MeV}$ \\
$M_{\pi^{0}}$ & $938.272 \mathrm{MeV}$ \\
$m_{p}$ & $939.565 \mathrm{MeV}$ \\
$m_{n}$ & $2.05(30) \mathrm{MeV}^{-}$ \\
$\delta m^{\text {QCD }}$, Ref. [6] & $1 / 137.036$ \\
\hline Fine-structure constant $\alpha:$ & $92.2 \mathrm{MeV}^{-}$ \\
\hline Pion decay constant $F_{\pi}:$ & 1.289 \\
\hline Effective axial-vector coupling of the nucleon $g_{A}^{\text {eff: }}:$ & $-1.10(3) \mathrm{GeV}^{-1}$ \\
\hline Pion-nucleon LECs from Ref. [5]: & $3.57(4) \mathrm{GeV}^{-1}$ \\
$c_{1}$ & $-5.54(6) \mathrm{GeV}^{-1}$ \\
$c_{2}$ & $4.17(4) \mathrm{GeV}^{-1}$ \\
$c_{3}$ & $6.18(8) \mathrm{GeV}^{-2}$ \\
$c_{4}$ & $-8.91(9) \mathrm{GeV}^{-2}$ \\
$\bar{d}_{1}+\bar{d}_{2}$ & $0.86(5) \mathrm{GeV}^{-2}$ \\
$\bar{d}_{3}$ & $-12.18(12) \mathrm{GeV}^{-2}$ \\
$\bar{d}_{5}$ & $1.18(4) \mathrm{GeV}^{-3}$ \\
$\bar{d}_{14}-\bar{d}_{15}$ & $-0.18(6) \mathrm{GeV}^{-3}$ \\
$\bar{e}_{14}$ & \\
$\bar{e}_{17}$ &
\end{tabular}

$g_{A}$ in all expressions with a rescaled effective axial vector coupling $g_{A}^{\text {eff }}$. For consistency reasons, we employ the values for $F_{\pi}$ and $g_{A}^{\text {eff }}$ from the determination of the $\pi \mathrm{N}$ LECs in Ref. [5]. For the QCD contribution to the nucleon mass difference, we use the value $\delta m^{\mathrm{QCD}}=2.05(30) \mathrm{MeV}$ [6] which is compatible with both the lattice QCD and QED result of Ref. [7] obtained by using the experimental value of $\delta m, \delta m^{\mathrm{QCD}} \simeq 2.16 \mathrm{MeV}$, and with the recent determination by the same authors $\delta m^{\mathrm{QCD}} \simeq 1.87(16) \mathrm{MeV}[8]$. The short-range part of the potential involves 25 isospin-invariant contact interactions, see Eqs. (A.1) and (17) of Ref. [1], and 5 IB contact terms as explained above. The strengths $C_{i}(\Lambda)$ of the corresponding interactions are treated as free parameters.

The NN potential specified above defines our central model (Model 1) used in the determination of $f^{2}$. To estimate the systematic uncertainty from the truncation of the EFT expansion of the IB NN potential, we also consider two additional interaction models that include selected IB contributions at sixth order (i.e. $\mathrm{N}^{5} \mathrm{LO}$ ):

Model 2: Same as Model 1 but including the subleading $\pi \gamma$-exchange potential driven by the numerically large isovector magnetic moment $\kappa_{v} \simeq 4.706$ of the nucleon, which has been worked out in Ref. [9].

Model 3: Same as Model 1 but including the IB TPE potential $\propto\left(f_{j}-f_{k}\right) c_{i}$. These contributions have not been considered before. The unregularized expressions read:

$$
\begin{aligned}
& V_{T}^{(6)}(q)=-\frac{1}{q^{2}} V_{S}^{(6)}(q)=-\frac{f_{\mathrm{c}}\left(2 f_{\mathrm{c}}-f_{\mathrm{p}}-f_{\mathrm{n}}\right) c_{4}}{4 F_{\pi}^{2} M_{\pi^{ \pm}}^{2}}\left(4 M_{\pi}^{2}+q^{2}\right) A(q), \\
& W_{C}^{(6)}(q)=-\frac{f_{\mathrm{c}}\left(f_{\mathrm{p}}-f_{\mathrm{n}}\right)}{2 F_{\pi}^{2} M_{\pi^{ \pm}}^{2}}\left(2 M_{\pi}^{2}+q^{2}\right)\left(4 M_{\pi}^{2} c_{1}-c_{3}\left(2 M_{\pi}^{2}+q^{2}\right)\right) A(q),
\end{aligned}
$$

where the function $A(q)$ is given by $A(q)=1 /(2 q) \arctan \left(q /\left(2 M_{\pi}\right)\right)$. 


\section{Calculation of the scattering amplitude in the presence of electromagnetic interactions}

In addition to the finite-range NN force described above, it is necessary to take into account the long-range electromagnetic interactions when calculating the scattering amplitude. Throughout this work, we include the so-called improved Coulomb potential [10], the magnetic moment interaction [11] as well as the vacuum-polarization potential [12] as described in detail in Ref. [1]. These effects have also been taken into account in the PWAs by the Nijmegen [13] and Granada [14, 15] groups. The nuclear amplitude is calculated by solving the Lippmann-Schwinger equation in the partial wave basis including all channels up to $j=20$.

\section{Objective function}

To determine the values of $f_{\mathrm{p}}^{2}, f_{0}^{2}$ and $f_{\mathrm{c}}^{2}$ from np and pp scattering data, we employ the same augmented $\chi^{2}$ measure as in Ref. [1]. Specifically, for the $j^{\text {th }}$ experiment consisting of $n_{j}$ measurements $\left\{O_{j, i}^{\exp }\right\}_{i=1, \ldots, n_{j}}$ of some observable and their corresponding statistical errors $\left\{\delta O_{j, i}^{\exp }\right\}_{i=1, \ldots, n_{j}}$, we define its $\chi^{2}$ measure as

$$
\chi_{j}^{2}=\sum_{i=1}^{n_{j}}\left(\frac{O_{j, i}^{\exp }-Z_{j} O_{j, i}^{\text {theo }}}{\delta O_{j, i}}\right)^{2}+\left(\frac{Z_{j}-1}{\delta_{\text {norm }, j}}\right)^{2} .
$$

Here, $\left\{O_{j, i}^{\text {theo }}\right\}_{i=1, \ldots, n_{j}}$ are the theoretical values of the observable calculated from the NN potential and $\delta_{\text {norm, } \mathrm{j}}$ is the (systematic) normalization error of the dataset $j$. If $\delta_{\text {norm, } \mathrm{j}} \neq 0$, the optimal norm is estimated by minimizing $\chi_{j}^{2}$ with respect to the factor $Z_{j}$, see Ref. [1] for further information. Our total $\chi^{2}$ is the sum of the contributions of np and pp scattering data in a given energy range and two additional data points for the deuteron binding energy $B_{d}$ and the np coherent scattering length $b_{\mathrm{np}}$ :

$$
\chi^{2}=\sum_{j} \chi_{j}^{2}+\left(\frac{B_{d}-B_{d}^{\exp }}{\delta B_{d}}\right)^{2}+\left(\frac{b_{\mathrm{np}}-b_{\mathrm{np}}^{\exp }}{\delta b_{\mathrm{np}}}\right)^{2},
$$

with $B_{d}^{\exp }=2.224575(9) \mathrm{MeV}$ [16] and $b_{\mathrm{np}}^{\exp }=-3.7405(9)$ fm [17]. Following Ref. [1], we use a slightly relaxed error for $B_{d}, \delta B_{d}=5 \times 10^{-5} \mathrm{MeV}$, and employ the experimental uncertainty of $b_{\mathrm{np}}$ for $\delta b_{\mathrm{np}}$.

\section{Data selection}

Following the same criteria as Refs. [13, 14, 18], we only consider NN data published in peer-reviewed journals and do not take into account $\mathrm{pp}$ total cross sections or quasi-elastic scattering data, i.e. data that have been extracted from three-body experiments. It is well known that not all available NN scattering data are mutually compatible within statistical errors. To determine mutually compatible np and pp data, we follow the standard iterative procedure to reject $3 \sigma$-inconsistent data [14, 15, 18]. Specifically, we start from a fit to all considered NN data which yields $\chi^{2} /$ datum $>1$. If the residuals of the objective function are properly normal-distributed, then $\chi_{j}^{2}$ of Eq. S6] should follow a $\chi^{2}$-distribution with $n_{j}$ degrees-of-freedom $\left(n_{j}-1\right.$ for floated datasets, see Ref. [1]). If for some dataset $\chi_{j}^{2}$ is either too high or too low, i.e. if the probability of obtaining that value is $\leq 0.27 \%$, the dataset is rejected. The potential is refitted to the accepted data and the compatibility of all data is tested again, resulting in a new set of accepted datasets. This process is then repeated until the data selection has reached stability.

For the selection process, simultaneous fits of all contact LECs and the $\pi \mathrm{N}$ constants $f_{\mathrm{p}}^{2}, f_{0}^{2}$ and $f_{\mathrm{c}}^{2}$ were performed using the central model (Model 1). We used a fixed cutoff value of $\Lambda=450 \mathrm{MeV}$ which was shown in Ref. [1] to yield the best description of $\mathrm{NN}$ data among the considered cutoffs and whose choice is verified a posteriori by being close to the optimal value $\Lambda=463.5 \mathrm{MeV}$ of this analysis. For each value of $E_{\text {lab }}^{\max }$ employed in this work, we have performed a separate data selection, and we found consistent results for $E_{\text {lab }}^{\max }=260-300 \mathrm{MeV}$. Our data selection is largely in agreement with the Granada 2013 database from Ref. [14] and, for the sake of brevity, we restrict ourselves to listing differences between our data selection at $E_{\mathrm{lab}}^{\max }=300 \mathrm{MeV}$ and the Granada 2013 database in Tables $\mathbf{S 2}$ and S3. As mentioned above, both tables also give our database for $E_{\mathrm{lab}}^{\max }=260$ and $280 \mathrm{MeV}$ if restricted to the corresponding energy ranges. For pp data with $E_{\mathrm{lab}}^{\max } \leq 240 \mathrm{MeV}$, the 3 differential cross sections of PA(58) get rejected, while the 15 differential cross sections of PA(58) and $\mathrm{CO}(67)$ rejected at higher energies are compatible with the rest of the data based on the $3 \sigma$ criterion. The $\mathrm{CO}(67)$ dataset, in particular, consists of measurements of remarkable precision that are sensitive to F-waves, see Ref. [1] for details. While it is properly described by our potential within estimated truncation errors for all $E_{\text {lab }}^{\max }$, its $\chi^{2}$ value is slightly too high to be statistically accepted 
TABLE S2. Differences in the np data selection of this work with $E_{\max }=300 \mathrm{MeV}$ in comparison to the Granada 2013 database [14]

\begin{tabular}{|c|c|c|c|c|c|}
\hline$E_{\text {lab }}[\mathrm{MeV}]$ & Ref. Code & Obs. & $n$ & comment & Ref. \\
\hline \multicolumn{6}{|c|}{ additionally included data } \\
\hline $0.509-2.003$ & $\mathrm{PO}(82)$ & $\sigma_{\text {tot }}$ & 3 & & [20] \\
\hline $1.005-2.530$ & $\mathrm{FI}(54)$ & $\sigma_{\text {tot }}$ & 2 & & 21 \\
\hline 2.535 & $\mathrm{DV}(71)$ & $\sigma_{\text {tot }}$ & 1 & & 22] \\
\hline 25.8 & $\mathrm{OC}(91)$ & $D_{t}$ & 1 & (a) & 23] \\
\hline 77.0 & WH(60) & $P$ & 8 & (a) & [24] \\
\hline 77.0 & WH(60) & $P$ & 9 & (a) & [24] \\
\hline 199.0 & $\mathrm{TH}(68)$ & $P$ & 8 & & 25 \\
\hline 200.0 & $\mathrm{KA}(63)$ & $d \sigma / d \Omega$ & 19 & (b) & 26] \\
\hline 300.0 & $\mathrm{DE}(54)$ & $d \sigma / d \Omega$ & 15 & & 27 \\
\hline \multicolumn{6}{|c|}{ additionally rejected data } \\
\hline 0 & $\mathrm{LO}(74)$ & $\sigma_{\text {tot }}$ & 1 & (c) & 28 \\
\hline $0.155-0.795$ & $\mathrm{DA}(13)$ & $\sigma_{\text {tot }}$ & 65 & & 29] \\
\hline 22.5 & $\mathrm{FL}(62)$ & $\sigma_{\text {tot }}$ & 1 & (d) & 30 \\
\hline 50.0 & $\mathrm{FI}(80)$ & $P$ & 4 & & 31 \\
\hline 135.0 & $\mathrm{LE}(63)$ & $A$ & 5 & (e) & 32 \\
\hline 137.0 & $\mathrm{LE}(63)$ & $R$ & 5 & (e) & 32 \\
\hline 197.0 & $\mathrm{SP}(67)$ & $D_{t}$ & 3 & (e) & 33 \\
\hline 295.0 & GR(82) & $\sigma_{\mathrm{tot}}, \Delta \sigma_{\mathrm{T}}, \Delta \sigma_{\mathrm{L}}$ & 3 & (f) & [34 \\
\hline
\end{tabular}
(a) data not considered in Granada database selection process
(b) the outlier at $97.0^{\circ}$ has been removed due to too high individual $\chi^{2}$
(c) $\mathrm{LO}(74)$ cites the data of Ref. [35], which is already included in the database
(d) the original publication explicitly mentions that no total cross section has been measured
(e) data from quasi-elastic scattering
(f) dispersion relation prediction

for $E_{\text {lab }}^{\max } \geq 260 \mathrm{MeV}$. For np data with $E_{\text {lab }}^{\max } \leq 240 \mathrm{MeV}, \mathrm{KA}(63)$ gets rejected, and the dataset of Ref. [19] is accepted for $E_{\text {lab }}^{\max }=240 \mathrm{MeV}$ only.

In addition, we have corrected minor misprints in Ref. [14] for the data of Refs. [30, 41] and removed erroneously assigned normalization errors $\delta_{\text {norm }, \mathrm{j}}$ to the datasets of Refs. [23, 42, 43] which only contain a single data point.

\section{Calculation of the marginalized PDF $p\left(f^{2} \mid D\right)$}

In our Bayesian analysis, we employ an uninformative uniform prior for the cutoff $\Lambda$, namely $p(\Lambda)=\left(\Lambda_{\max }-\Lambda_{\min }\right)^{-1}$ for $\Lambda \in\left[\Lambda_{\min }, \Lambda_{\max }\right]$, while 0 elsewhere. Similarly, we use a uniform prior for the $\pi \mathrm{N}$ coupling constants $f_{i}^{2}$, which is non-zero in a sufficiently large region $B=\left\{f^{2} \mid f_{i, \min }^{2} \leq f_{i}^{2} \leq f_{i, \max }^{2}\right\}$ around their previously obtained values. Finally, we employ a Gaussian prior for the LECs $C_{i}, p(C)=(\sqrt{2 \pi} \bar{C})^{-n} \exp \left(-\vec{C}^{2} /\left(2 \bar{C}^{2}\right)\right)$, with $n=30$ being the dimension of $\vec{C}$, to encode the naturalness assumptions for their values. Here, the parameter $\bar{C}$ to be specified below is dimension-less, and the (dimensionful) $C_{i}$ 's in the spectroscopic notation are expressed in their natural units of $4 \pi /\left(F_{\pi}^{2} \Lambda_{\mathrm{b}}^{2 k}\right)$ with $2 k$ being the power of momenta and $\Lambda_{\mathrm{b}}=650 \mathrm{MeV}$, see Ref. [1].

Using the approximation for the likelihood $p\left(D \mid f^{2} C \Lambda\right)$ in Eq. (3) of the main text and the explicit form of the priors $p(\Lambda)$, $p\left(f^{2}\right)$ and $p(C)$, the expression for the marginalized posterior in Eq. (2) of the main text takes the form

$$
p\left(f^{2} \mid D\right)=\frac{1}{\tilde{N}} \int_{\Lambda_{\min }}^{\Lambda_{\max }} d \Lambda \frac{1}{\sqrt{\operatorname{det} A}} e^{-\frac{1}{2}\left(\chi_{\min }^{2}+\frac{1}{C^{2}} C_{\min }^{T} C_{\min }-\frac{1}{\bar{C}^{4}} C_{\min }^{T} A^{-1} C_{\min }\right)} \mathbb{1}_{B}\left(f^{2}\right),
$$


TABLE S3. Same as Table S2 but for pp data

\begin{tabular}{llllll}
\hline$E_{\text {lab }}[\mathrm{MeV}]$ & Ref. Code & Obs. & $n$ & comment & Ref. \\
\hline \multicolumn{2}{l}{ additionally included data } & & & & \\
137.0 & $\mathrm{PA}(58)$ & $d \sigma / d \Omega$ & 3 & & [36] \\
239.9 & $\mathrm{AL}(04)$ & $d \sigma / d \Omega$ & 17 & $(\mathrm{a})$ & [37] \\
255.2 & $\mathrm{AL}(04)$ & $d \sigma / d \Omega$ & 18 & $(\mathrm{a})$ & (a) \\
270.8 & $\mathrm{AL}(04)$ & $d \sigma / d \Omega$ & 19 & & [37] \\
286.7 & $\mathrm{AL}(04)$ & $d \sigma / d \Omega$ & 20 & & [37] \\
\hline additionally rejected data & & & & & [39] \\
144.0 & $\mathrm{JA}(71)$ & $d \sigma / d \Omega$ & 27 & & [36] \\
144.1 & $\mathrm{CO}(67)$ & $d \sigma / d \Omega$ & 15 & & [40] \\
147.0 & $\mathrm{PA}(58)$ & $d \sigma / d \Omega$ & 15 & & \\
217.0 & $\mathrm{TI}(61)$ & $P$ & 6 & & \\
\hline
\end{tabular}

where $A=\frac{1}{2} H+\frac{1}{\bar{C}^{2}} \mathbb{1}$, the normalization constant $\tilde{N}$ is given by $\tilde{N}=N \bar{C}^{n} p(D)\left(\Lambda_{\max }-\Lambda_{\min }\right) \prod_{i}\left(f_{i, \max }^{2}-f_{i, \min }^{2}\right)$ and $\mathbb{1}_{B}$ is the indicator function of the cuboid $B$.

It remains to specify the parameters entering the priors $p(\Lambda), p\left(f^{2}\right)$ and $p(C)$. For the naturalness prior of the short-range LECs $C_{i}$ we choose the value of $\bar{C}=5$. Given the abundance of NN scattering data, the $C_{i}$ 's are well-constrained by the likelihood. In our previous study [1], the $C_{i}$ 's were found to be of natural size in the considered cutoff range of $\Lambda$, i.e. $\left|C_{i}\right| \sim 1$, even without imposing any naturalness constraints. In particular, none of the values of $\left|C_{i}\right|$ from Ref. [1] exceed $\bar{C}=5$, see Fig. 7 of Ref. [44]. We, therefore, expect the naturalness constraint $\bar{C}=5$ to have a negligible effect on the obtained results. The same Gaussian prior with $\bar{C}=5$ was also employed in Ref. [45] in a fit to scattering phase shifts and was reported to have only minor impact. Next, for the $p(\Lambda)$-prior, we employ the values of $\Lambda_{\min }=400 \mathrm{MeV}$ and $\Lambda_{\max }=550 \mathrm{MeV}$. Figure S1 shows, as a representative example, the integrand of Eq. (S8) at the central values for the $f_{i}^{2}$ 's for the case of Model 1 and $E_{\mathrm{lab}}=0-280 \mathrm{MeV}$ in the employed cutoff range. The maximum of the integrand is at $\Lambda \simeq 463.5 \mathrm{MeV}$, and its contribution beyond $\Lambda=430-510 \mathrm{MeV}$ has already dropped by more than five orders of magnitude compared to its maximum and is negligible. Thus, the cutoff is constrained by the likelihood and not by the prior. We emphasize that the preference of the $\Lambda=430-510 \mathrm{MeV}$ region does not contradict the findings of Ref. [1], where a weak $\Lambda$-dependence of the $\chi^{2} /$ datum values was shown. Indeed, $\chi^{2}$ values have a logarithmic scale compared to the probability density and thus vary much less. Finally, for the uniform $p\left(f^{2}\right)$-prior, we choose the parameters to be $f_{\mathrm{p}, \min }^{2}=f_{0, \min }^{2}=f_{\mathrm{c}, \min }^{2}=0.0729, f_{\mathrm{p}, \max }^{2}=f_{\mathrm{c}, \max }^{2}=0.0812$ and $f_{0, \max }^{2}=0.0827$. The chosen limits of $f_{i}^{2}$ only enter the marginal PDF, and the adequacy of their choice becomes obvious from Fig. 2 of the main text. To summarize, we expect that making the priors $p(\Lambda), p\left(f^{2}\right)$ and $p(C)$ less informative should have a negligible effect on our results.

The remaining integration over $\Lambda$ in Eq. $(S 8)$ is performed numerically via Gaussian quadrature. Scaling up the number of integration points for the cutoff naively is computationally costly as each evaluation of the integrand in Eq. (S8) requires a fit of 30 contact LECs. However, the logarithm of the integrand is a smooth function so that we can safely evaluate it at just 8 points in the range of $\Lambda=400-550 \mathrm{MeV}$ and interpolate it by a polynomial.

\section{Systematic uncertainty due to the choice of the energy range in the fits and neglect of IB interactions beyond $\mathrm{N}^{4} \mathrm{LO}$}

To extract the constants $f^{2}$ from NN data, one needs to specify the maximum energy of data considered in the PWA $E_{\text {lab }}^{\max }$. To stay unbiased, we perform determinations of $f^{2}$ 's at different values of $E_{\mathrm{lab}}^{\max }$ varied in steps of $20 \mathrm{MeV}$. Since our theoretical framework and the employed parametrization of the scattering amplitude are valid below the pion production threshold, the largest value of $E_{\text {lab }}^{\max }$ we consider is $E_{\text {lab }}^{\max }=300 \mathrm{MeV}$. On the other hand, when lowering $E_{\text {lab }}^{\max }$, the number of experimental data gets reduced leading to larger statistical errors, and one runs the danger of overfitting. To verify consistency of our fit results at individual energies, we compare the resulting quantiles of the empirical distribution of residuals against the ones of the assumed normal distribution $\mathcal{N}(0,1)$. In order to statistically quantify deviations from the Gaussian distribution of residuals, various confidence bands have been derived in the literature. In this work we employ the ones by Aldor-Noiman et al. [46] which are one of the most recent and most sensitive, especially towards the tails of the quantile-quantile plot. In Fig. S2 b, one observes that the corresponding graphical normality test is fulfilled at the $1 \sigma$-level for the fit up to $E_{\text {lab }}^{\max }=300 \mathrm{MeV}$. When the energy 


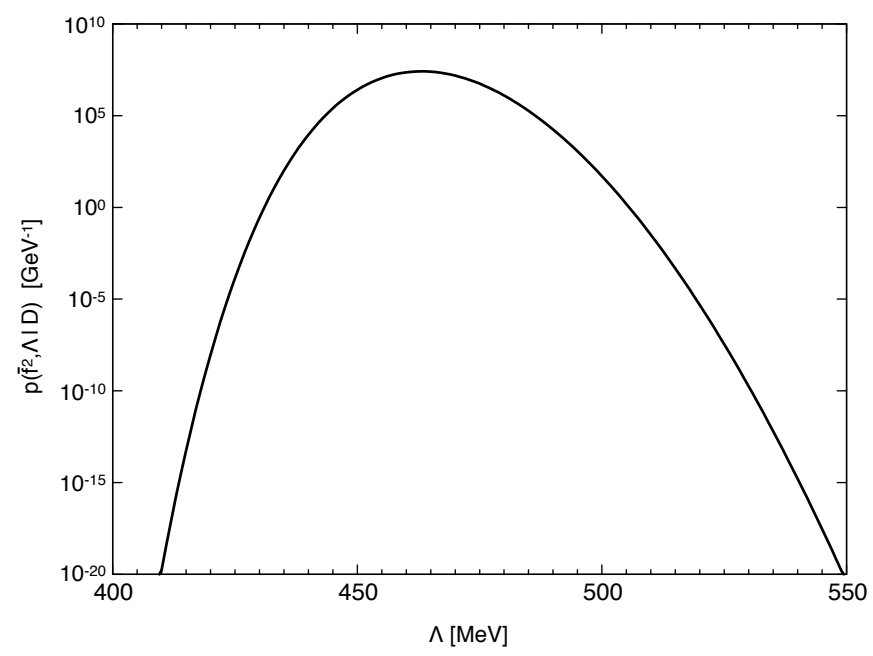

FIG. S1. The integrand of Eq. $\left[\mathrm{S} 8\right.$, as a function of the cutoff $\Lambda$ for Model 1. $\bar{f}_{i}^{2}$ refer to the central values of the LECs $f_{i}^{2}$ given in Eq. (4) of the main text. The data $D$ correspond to the mutually compatible NN scattering data according to the own selection in the range of $E_{\text {lab }}=0-280 \mathrm{MeV}$.
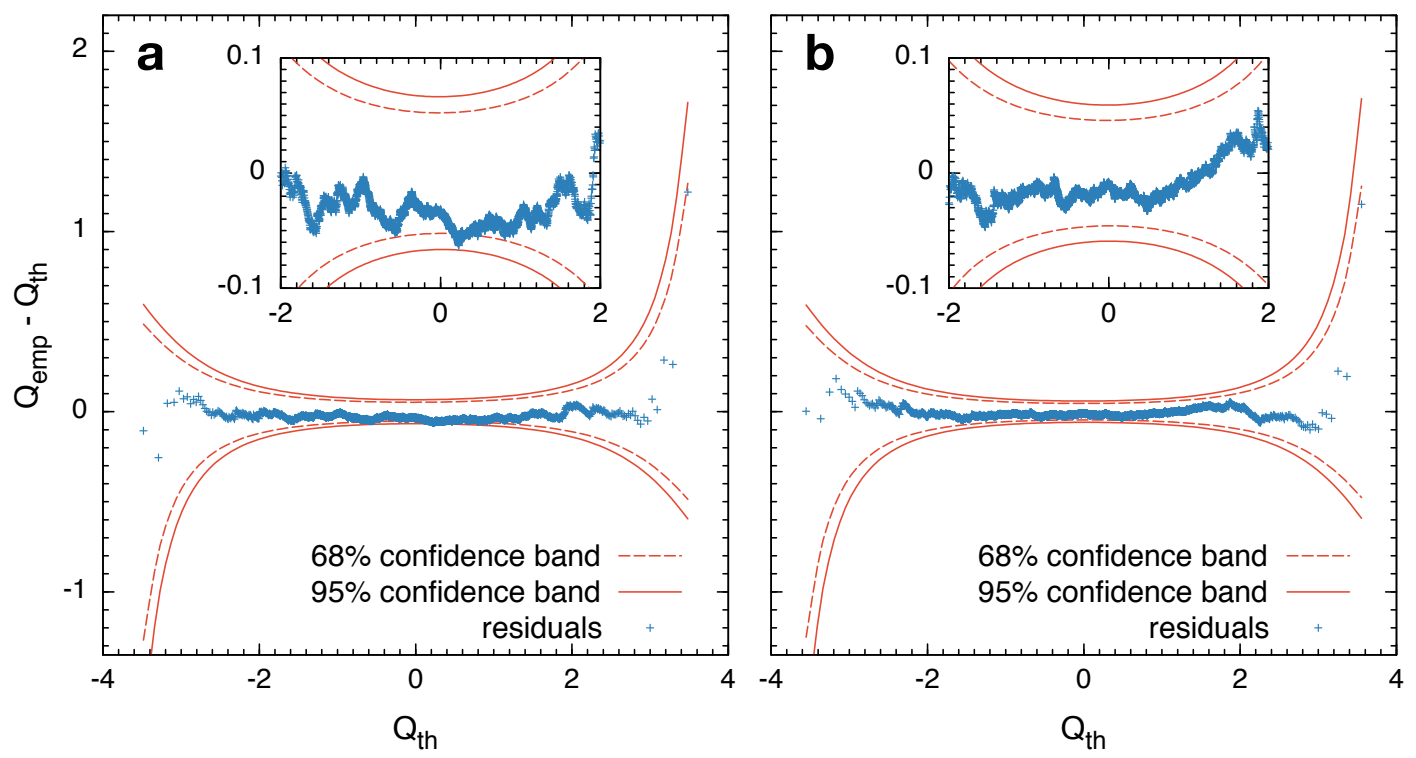

FIG. S2. Tail-sensitive rotated quantile-quantile plots for Model 1 and $\Lambda=463.5 \mathrm{MeV}$. The plots a and $\mathbf{b}$ show the results of the fits in the energy range of $E_{\text {lab }}=0-220 \mathrm{MeV}$ and $E_{\text {lab }}=0-300 \mathrm{MeV}$, respectively. The distribution of residuals, shown by blue crosses, is consistent with the normal distribution $\mathcal{N}(0,1)$ at a given confidence level if all points lie inside the corresponding confidence band. Dashed and solid lines mark the $68 \%$ and $95 \%$ confidence bands, respectively.

range of the fit is reduced to $\sim 200 \mathrm{MeV}$, we start observing systematic distortions from the normal distribution of residuals pointing towards a possible overfitting issue. We, therefore, do not take into account fits with $E_{\text {lab }}^{\max }$ below $E_{\text {lab }}^{\max }=220 \mathrm{MeV}$. For this energy range, the distribution of empirical residuals is still statistically consistent with the normal one at the $2 \sigma$-level, see Fig. S2a. This leaves us with five energy ranges corresponding to $E_{\text {lab }}^{\max }=220,240,260,280$ and $300 \mathrm{MeV}$.

In Table S4, we show the extracted values of the constants $f^{2}$ for three different interaction models and five energy ranges. In each case, we have determined the maximum of the marginal posteriors $p\left(f^{2} \mid D\right)$ using the mutually consistent $\mathrm{np}$ and $\mathrm{pp}$ data from the independent data selection up to the given value of $E_{\mathrm{lab}}^{\max }$ for Models 1,2 and 3 by numerically evaluating the integral in Eq. [S8). Around 50 evaluations of the marginal posterior turn out to be sufficient to accurately determine the maximum of $p\left(f^{2} \mid D\right)$. For the employed grid of $8 \Lambda$-values, we thus had to carry out $\sim 400$ determinations of the set of 30 LECs $C$ for every of the 15 considered cases.

While the central values of the $f^{2}$ can be conveniently determined in this way, numerically obtaining the full marginal poste- 
TABLE S4. Central values and statistical errors of the $\pi \mathrm{N}$ coupling constants $f_{i}^{2}$ for all considered interaction models and $E_{\mathrm{lab}}^{\mathrm{max}}$ values

\begin{tabular}{|c|c|c|c|c|c|}
\hline & $220 \mathrm{MeV}$ & $240 \mathrm{MeV}$ & $260 \mathrm{MeV}$ & $280 \mathrm{MeV}$ & $300 \mathrm{MeV}$ \\
\hline \multicolumn{6}{|l|}{ Model 1: } \\
\hline$f_{\mathrm{p}}^{2}$ & $0.0772(5)$ & $0.0772(5)$ & $0.0769(4)$ & $0.0768(4)$ & $0.0769(4)$ \\
\hline$f_{0}^{2}$ & $0.0782(10)$ & $0.0783(9)$ & $0.0781(8)$ & $0.0780(8)$ & $0.0782(8)$ \\
\hline$f_{\mathrm{c}}^{2}$ & $0.0770(5)$ & $0.0770(5)$ & $0.0769(4)$ & $0.0767(4)$ & $0.0766(4)$ \\
\hline \multicolumn{6}{|l|}{ Model 2: } \\
\hline$f_{\mathrm{p}}^{2}$ & $0.0772(5)$ & $0.0772(5)$ & $0.0769(4)$ & $0.0768(4)$ & $0.0769(4)$ \\
\hline$f_{0}^{2}$ & $0.0782(9)$ & $0.0782(9)$ & $0.0780(8)$ & $0.0779(8)$ & $0.0782(8)$ \\
\hline$f_{\mathrm{c}}^{2}$ & $0.0771(5)$ & $0.0771(5)$ & $0.0770(4)$ & $0.0767(4)$ & $0.0767(4)$ \\
\hline \multicolumn{6}{|l|}{ Model 3: } \\
\hline$f_{\mathrm{p}}^{2}$ & $0.0772(5)$ & $0.0772(5)$ & $0.0770(4)$ & $0.0768(4)$ & $0.0770(4)$ \\
\hline$f_{0}^{2}$ & $0.0778(9)$ & $0.0779(9)$ & $0.0776(8)$ & $0.0775(7)$ & $0.0777(7)$ \\
\hline$f_{\mathrm{c}}^{2}$ & $0.0772(5)$ & $0.0771(5)$ & $0.0771(4)$ & $0.0768(4)$ & $0.0768(4)$ \\
\hline
\end{tabular}

riors on a sufficiently dense grid is computationally costly, and it was only done for our central model with $E_{\text {lab }}^{\max }=280 \mathrm{MeV}$ as shown in Fig. 2 of the main text, This required performing 8000 fits of the contact LECs $C$. As discussed in the text, the marginal posterior can be very well approximated by a multivariate Gaussian distribution, whose central values are given by the maximum of $p\left(f^{2} \mid D\right)$ and whose covariance matrix can be inexpensively calculated from the $\sim 50$ points evaluated during the determination of the maximum. The covariance matrices then yield the uncertainties quoted in Table $\mathrm{S} 4$ and, for the sake of completeness, we also give the corresponding correlation coefficients in Table S5 In order to arrive at our final recommended values for the $\pi \mathrm{N}$ couplings $f_{i}^{2}$, we perform a simple averaging of the 15 multivariate Gaussian distributions obtained above

$$
\begin{aligned}
p_{\text {avg }}\left(f^{2} \mid \bar{D}\right) & =\frac{1}{5} \sum_{i, j} p\left(f^{2} \mid D_{i} M_{j}\right) p\left(M_{j} \mid D_{i}\right), \\
& \approx \frac{1}{15} \sum_{i, j} p\left(f^{2} \mid D_{i} M_{j}\right),
\end{aligned}
$$

where $p\left(f^{2} \mid D_{i} M_{j}\right)$ is given by Eq. S8 8 with $D_{i} \equiv D\left(E_{\text {lab,i }}^{\max }\right)$ being the selected data for $E_{\text {lab }}^{\max }=220,240,260,280,300 \mathrm{MeV}$ and $\left\{M_{j}\right\}$ is the set of the three potential models. The $p\left(M_{j} \mid D_{i}\right)$ are the model weights for the different models and different values of $E_{\text {lab }}^{\max }$. Since the description of the data is approximately equal among the three models, we have set the $p\left(M_{j} \mid D_{i}\right)=$ $1 / 3$ to be equal as well in the second line of Eq. $\left[\mathbf{S 9}\right.$ ). Furthermore, $\bar{D}$ denotes the dependence on the data averaged over $E_{\text {lab }}^{\max }$. In the simple averaging of Eq. [S9] all combinations of Models and $E_{\text {lab }}^{\max }$ enter with the same weight. The straightforward averaging over the data for different $E_{\text {lab }}^{\max }$ reflects that all values of $E_{\text {lab }}^{\max }$ are to be treated equally on statistical grounds. Since the maximum of $p\left(f^{2} \mid D_{i} M_{j}\right)$ gets more localized in the presence of more data (i.e. for higher values of $\left.E_{\mathrm{lab}}^{\max }\right)$ and simultaneously larger in magnitude (due to the normalization of $p\left(f^{2} \mid D_{i} M_{j}\right)$ ), there is a natural weighting towards the posteriors including more data. The development of a formal Bayesian model for the data averaging over $E_{\text {lab }}^{\max }$ goes beyond the scope of the present study. Given the rather stable results for the coupling constants $f_{i}^{2}$ with respect to the variation of $E_{\mathrm{lab}}^{\max }$, see Tables S4 and S5, we expect the employed procedure for averaging over $E_{\text {lab }}^{\max }$ to be sufficient for the purpose of this study. The final results for the central values and errors (a) of the $f_{i}^{2}$ given in Eq. (4) of the main text have been obtained by approximating $p_{\text {avg }}\left(f^{2} \mid D\right)$ with a single multivariate Gaussian around its maximum.

\section{Verification of Bayesian model weights}

In the averaging of Eq. $S 9$ ) used to obtain our recommended values of the pion-nucleon coupling constants, we have set the model weights $p\left(M_{j} \mid D_{i}\right)$ to be equal. In order to verify this approximation, we have repeated the model averaging with the exact Bayesian model weights

$$
p\left(M_{j} \mid D_{i}\right)=\frac{p\left(D_{i} \mid M_{j}\right) p\left(M_{j}\right)}{\sum_{k} p\left(D_{i} \mid M_{k}\right) p\left(M_{k}\right)} \rightarrow \frac{p\left(D_{i} \mid M_{j}\right)}{\sum_{k} p\left(D_{i} \mid M_{k}\right)},
$$


TABLE S5. Correlation coefficients between $f_{i}^{2}$, s for all considered interaction models and $E_{\text {lab }}^{\max }$ values

\begin{tabular}{|c|c|c|c|c|c|}
\hline & $220 \mathrm{MeV}$ & $240 \mathrm{MeV}$ & $260 \mathrm{MeV}$ & $280 \mathrm{MeV}$ & $300 \mathrm{MeV}$ \\
\hline \multicolumn{6}{|l|}{ Model 1: } \\
\hline $\operatorname{corr}\left(f_{\mathrm{p}}^{2}, f_{0}^{2}\right)$ & -0.05 & -0.04 & -0.01 & -0.04 & -0.04 \\
\hline $\operatorname{corr}\left(f_{0}^{2}, f_{\mathrm{c}}^{2}\right)$ & -0.13 & -0.08 & -0.12 & -0.14 & -0.15 \\
\hline $\operatorname{corr}\left(f_{\mathrm{c}}^{2}, f_{\mathrm{p}}^{2}\right)$ & 0.32 & 0.28 & 0.35 & 0.28 & 0.27 \\
\hline \multicolumn{6}{|l|}{ Model 2: } \\
\hline $\operatorname{corr}\left(f_{\mathrm{p}}^{2}, f_{0}^{2}\right)$ & -0.04 & -0.04 & -0.01 & -0.04 & -0.04 \\
\hline $\operatorname{corr}\left(f_{0}^{2}, f_{\mathrm{c}}^{2}\right)$ & -0.10 & -0.09 & -0.12 & -0.14 & -0.12 \\
\hline $\operatorname{corr}\left(f_{\mathrm{c}}^{2}, f_{\mathrm{p}}^{2}\right)$ & 0.29 & 0.29 & 0.35 & 0.28 & 0.27 \\
\hline \multicolumn{6}{|l|}{ Model 3: } \\
\hline $\operatorname{corr}\left(f_{\mathrm{p}}^{2}, f_{0}^{2}\right)$ & 0.00 & 0.00 & 0.04 & 0.06 & 0.06 \\
\hline $\operatorname{corr}\left(f_{0}^{2}, f_{\mathrm{c}}^{2}\right)$ & -0.03 & -0.01 & -0.05 & -0.07 & -0.04 \\
\hline $\operatorname{corr}\left(f_{\mathrm{c}}^{2}, f_{\mathrm{p}}^{2}\right)$ & 0.29 & 0.28 & 0.33 & 0.28 & 0.25 \\
\hline
\end{tabular}

where $p\left(D_{i} \mid M_{j}\right)$ is the data posterior of the data $D_{i}$ for a particular model $M_{j}$ and $p\left(M_{j}\right)$ is the model prior. We choose a uniform model prior $p\left(M_{k}\right)=1 / N_{M}$ with $N_{M}=3$ the number of models, yielding the last expression in Eq. [S10]. The data posterior can be obtained by marginalizing over all parameters of the joint posterior

$$
p\left(D_{i} \mid M_{j}\right)=\int d f^{2} d \Lambda d C p\left(D_{i} \mid f^{2} C \Lambda M_{j}\right) p\left(f^{2} C \Lambda\right) .
$$

Eq. (S11) can be obtained from Eq. (2) of the main text where the dependence on the model $M_{j}$ had been notationally suppressed. In a frequentist analysis the model weights of Eq. S10 are qualitatively similar to a weighting with the exponential of the $\chi^{2}$ value of each model $M_{j}$. The model weights thus become important when the involved models do not describe the data equally well. Employing our Bayesian model weights $p\left(M_{j} \mid D_{i}\right)$ in the first line of Eq. $(\mathbf{S 9}$ ) and repeating the averaging, we obtain

$$
\begin{aligned}
& f_{\mathrm{p}}^{2}=0.0770(5)^{\mathrm{a}}, \\
& f_{0}^{2}=0.0780(9)^{\mathrm{a}}, \\
& f_{\mathrm{c}}^{2}=0.0768(5)^{\mathrm{a}},
\end{aligned}
$$

in good agreement with our recommended values of Eq. (4) of the main text.

\section{Sensitivity to the values of the higher-order $\pi$ N LECs}

To propagate the statistical errors in the values of the higher-order $\pi \mathrm{N}$ LECs $c_{i}, \bar{d}_{i}$ and $\bar{e}_{i}$ determined from the Roy-Steiner equation analysis of Ref. [5], see Table S1, we have generated a sample of 50 sets of normally distributed values of these LECs. For each set, the determination of $f^{2}$ is repeated for the central model (albeit with different values of the corresponding $\pi \mathrm{N}$ LECs each time) and $E_{\max }=280 \mathrm{MeV}$. The resulting uncertainties of the $f^{2}$ is then obtained by taking the standard deviation of the these 50 values of the $f^{2}$.

\section{Impact of scattering data after 1993}

In Fig. 3 of the main text we compare our results for the $\pi \mathrm{N}$ coupling constants to various other determinations of the last 30 years. Here, the results of the Nijmegen group [47, 48] and the Granada group [15] are of particular interest since they are also obtained from NN scattering data. As already pointed out in the main text, we find, similarly to the Granada 2017 analysis, significantly larger values of all coupling constants $f_{\mathrm{p}}^{2}, f_{0}^{2}$ and $f_{\mathrm{c}}^{2}$ compared to the Nijmegen results. The database of scattering data used by the Granada 2017 analysis is much more similar to ours than the older Nijmegen analyses of Refs. [47, 48] which 
evidently do not include many experimental data obtained after 1993. This raises the question if the larger values of the coupling constants are driven by the differences in databases.

In order to address this issue, we have repeated our analysis using scattering data up to 1993 for the Model 1 and $E_{\text {lab }}^{\max }=$ $280 \mathrm{MeV}$ To avoid possible overfitting issues due to a significantly smaller number of data and to facilitate a comparison with Ref. [15], we restrict ourselves to the average value of the coupling constant by setting $f_{p}^{2}=f_{c}^{2}=f_{0}^{2} \equiv f^{2}$ in the NN interaction. Performing a fit of the np and pp data using the complete 2020 database with $N_{\text {dat }}=4926$, we obtain the average value of the pion nucleon coupling constant of $f^{2}=0.07694(29)$ in a good agreement with the corresponding values quoted in Table $\mathrm{S4}$

We now repeat the determination by retaining only the experimental data available in 1993. The data selection using the $3 \sigma$-criterion is mostly consistent with our final 2020 database restricted to data before and including 1993 . Concerning the differences, we find that the proton-proton differential cross sections of Refs. [36, 38, 39] rejected in Table $[\mathrm{S3}$ are now included while the previously included dataset at $E_{\text {lab }}=137 \mathrm{MeV}$ of Ref. [36] is instead rejected. In the neutron-proton database, the dataset at $E_{\text {lab }}=96 \mathrm{MeV}$ of Ref. [49] is additionally rejected due to a too low $\chi^{2}$. Using this 1993 database with $N_{\text {dat }}=$ 3804 , we obtain essentially the same value of the coupling constant, $f^{2}=0.07698(35)$. Thus, according to our analysis, the significantly smaller value of $f^{2}$ found by the Nijmegen group cannot be explained by the scattering data published after 1993. For the sake of completeness, we mention that the Granada group found slightly smaller values of $f^{2}$ by excluding the data published after 1990 and 1995 from the Granada 2013 database 2 (without repeating the data selection), see Table X of Ref. [15], with the observed reduction in $f^{2}$ being, however, by far insufficient to explain the differences to the much smaller Nijmegen values.

[1] P. Reinert, H. Krebs, and E. Epelbaum, Eur. Phys. J. A 54, 86 (2018),

[2] U. van Kolck, M. C. M. Rentmeester, J. L. Friar, J. T. Goldman, and J. J. de Swart, Phys. Rev. Lett. 80, 4386 (1998)

[3] E. Epelbaum and U.-G. Meißner, Phys. Rev. C 72, 044001 (2005)

[4] E. Epelbaum, H. Krebs, and U.-G. Meißner, Phys. Rev. C 77, 034006 (2008)

[5] M. Hoferichter, J. Ruiz de Elvira, B. Kubis, and U.-G. Meißner, Phys. Rev. Lett. 115, 192301 (2015)

[6] J. Gasser and H. Leutwyler, Nucl. Phys. B 94, 269 (1975)

[7] S. Borsanyi et al., Science 347, 1452 (2015)

[8] J. Gasser, H. Leutwyler, and A. Rusetsky, Phys. Lett. B 814, 136087 (2021)

[9] N. Kaiser, Phys. Rev. C 73, 044001 (2006)

[10] G. J. M. Austen and J. J. de Swart, Phys. Rev. Lett. 50, 2039 (1983)

[11] V. G. J. Stoks and J. J. De Swart, Phys. Rev. C 42, 1235 (1990)

[12] L. Durand, Phys. Rev. 108, 1597 (1957)

[13] V. G. J. Stoks, R. A. M. Klomp, M. C. M. Rentmeester, and J. J. de Swart, Phys. Rev. C 48, 792 (1993)

[14] R. Navarro Pérez, J. E. Amaro, and E. Ruiz Arriola, Phys. Rev. C 88, 064002 (2013). [Erratum: Phys. Rev. C 91, 029901 (2015)].

[15] R. Navarro Pérez, J. E. Amaro, and E. Ruiz Arriola, Phys. Rev. C 95, 064001 (2017)

[16] C. van der Leun and C. Alderliesten, Nucl. Phys. A 380, 261 (1982)

[17] K. Schoen, D. L. Jacobson, M. Arif, P. R. Huffman, T. C. Black, W. M. Snow, S. K. Lamoreaux, H. Kaiser, and S. A. Werner, Phys. Rev. C 67, 044005 (2003)

[18] F. Gross and A. Stadler, Phys. Rev. C 78, 014005 (2008)

[19] D. F. Measday and J. N. Palmieri, Nucl. Phys. 85, 142 (1966)

[20] W. P. Poenitz and J. F. Whalen, Nucl. Phys. A 383, 224 (1982)

[21] D. Fischer and G. Goldhaber, Phys. Rev. 95, 1350 (1954)

[22] J. C. Davis, K. A. Weaver, D. Hilscher, H. H. Barschall, and A. B. Smith, Phys. Rev. C 4, 1061 (1971).

[23] M. Ockenfels, T. Köble, M. Schwindt, J. Weltz, and W. von Witsch, Nucl. Phys. A 534, 248 (1991)

[24] C. Whitehead, S. Tornabene, and G. H. Stafford, Proc. Phys. Soc. 75, 345 (1960)

[25] A. R. Thomas, D. Spalding, and E. H. Thorndike, Phys. Rev. 167, 1240 (1968)

[26] Y. M. Kazarinov and Y. N. Smirnov, Zh. Eksp. Teor. Fiz. 43, 35 (1962), [J. Exp. Theor. Phys. 16, 24 (1963)].

[27] J. de Pangher, Phys. Rev. 95, 578 (1954).

[28] E. Lomon and R. Wilson, Phys. Rev. C 9, 1329 (1974)

[29] B. H. Daub, V. Henzl, M. A. Kovash, J. L. Matthews, Z. W. Miller, K. Shoniyozov, and H. Yang, Phys. Rev. C 87, 014005 (2013)

[30] E. R. Flynn and P. J. Bendt, Phys. Rev. 128, 1268 (1962)

[31] D. H. Fitzgerald, F. P. Brady, R. Garrett, S. W. Johnsen, J. L. Romero, T. S. Subramanian, J. L. Ullmann, and J. W. Watson, Phys. Rev. C 21, $1190(1980)$

\footnotetext{
${ }^{1}$ We do keep, however, the np coherent scattering length of Ref. [17]. We have checked that this does not affect the conclusions of this section.

${ }^{2}$ Notice that both the Nijmegen and Granada groups also include scattering
}

data above the pion production threshold up to $E_{\text {lab }}=350 \mathrm{MeV}$ in their fits. 
[32] J. Lefrancois, R. A. Hoffman, E. H. Thorndike, and R. Wilson, Phys. Rev. 131, 1660 (1963),

[33] D. Spalding, A. R. Thomas, and E. H. Thorndike, Phys. Rev. 158, 1338 (1967)

[34] W. Grein and P. Kroll, Nucl. Phys. A 377, 505 (1982)

[35] T. L. Houk, Phys. Rev. C 3, 1886 (1971)

[36] J. N. Palmieri, A. M. Cormack, N. F. Ramsey, and R. Wilson, Ann. Phys. 5, 299 (1958).

[37] D. Albers et al., Eur. Phys. J. A 22, 125 (2004)

[38] O. N. Jarvis, C. Whitehead, and M. Shah, Phys. Lett. B 36, 409 (1971)

[39] G. F. Cox, G. H. Eaton, C. P. van Zyl, O. N. Jarvis, and B. Rose, Nucl. Phys. B 4, 353 (1968)

[40] J. H. Tinlot and R. E. Warner, Phys. Rev. 124, 890 (1961).

[41] G. Fink, P. Doll, T. D. Ford, R. Garrett, W. Heeringa, K. Hofmann, H. O. Klages, and H. Krupp, Nucl. Phys. A 518, 561 (1990)

[42] C. Amsler et al., Nucl. Instrum. Methods 144, 401 (1977)

[43] L. G. Greeniaus, D. A. Hutcheon, C. A. Miller, G. A. Moss, G. Roy, R. Dubois, C. Amsler, B. K. S. Koene, and B. T. Murdoch, Nucl. Phys. A 322, 308 (1979)

[44] E. Epelbaum, H. Krebs, and P. Reinert, Front. in Phys. 8, 98 (2020)

[45] S. Wesolowski, R. J. Furnstahl, J. A. Melendez, and D. R. Phillips, J. Phys. G 46, 045102 (2019).

[46] S. Aldor-Noiman, L. D. Brown, A. Buja, W. Rolke, and R. A. Stine, Am. Stat. 67, 249 (2013). [Erratum: Am. Stat. 68, 318 (2014)].

[47] V. G. J. Stoks, R. Timmermans, and J. J. de Swart, Phys. Rev. C 47, 512 (1993)

[48] R. A. M. Klomp, V. G. J. Stoks, and J. J. de Swart, Phys. Rev. C 44, R1258 (1991)

[49] T. C. Griffith, A. P. Banford, M. Y. Uppal, and W. S. C. Williams, Proc. Phys. Soc. 71, 305 (1958) 